\title{
Dynamical Systems Techniques for Designing Libration Point Orbits in Proximity of Highly-Inhomogeneous Planetary Satellites: Application to the Mars-Phobos Elliptic Three-Body Problem with Additional Gravity Harmonics
}

\author{
Mattia Zamaro and James D. Biggs \\ Advanced Space Concepts Laboratory, University of Strathclyde, Glasgow, United Kingdom
}

\begin{abstract}
The orbital dynamics around the Libration points of the classical circular restricted three-body problem (CR3BP) have been investigated in detail: in the last few decades, dynamical systems theory has provided invaluable analytical and numerical tools for understanding the dynamics of Libration Point Orbits (LPOs).

The aim of this paper is to extend the model of the CR3BP to derive the LPOs in the vicinity of the Martian moon Phobos, which is becoming an appealing destination for scientific missions. The case of Phobos is particularly extreme, since the combination of both small mass-ratio and length-scale moves the collinear Libration manifold close to the moon's surface. Thus, a model of this system must consider additional dynamical perturbations, in particular the complete gravity field of Phobos, which is highly-inhomogeneous. This is accomplished using a spherical harmonics series expansion, deriving an enhanced elliptic three-body model. In this paper, we show how methodologies from dynamical systems theory are applied in differential correction continuation schemes to this proposed nonlinear model of the dynamics near Phobos, to derive the structure of the dynamical substitutes of the LPOs in this new system.

Results obtained show that the structure of the LPOs differs substantially from the classical case without harmonics. The proposed methodology allows us to identify natural periodic and quasi-periodic orbits that would provide unique low-cost opportunities for close-range observations around Phobos and high-performance landing/take-off pathways to and from Phobos' surface, which could be exploited in upcoming missions targeting the exploration of this Martian moon.
\end{abstract}

Keywords: Phobos; LPO; Quasi-periodic; Gravity Harmonics; Dynamical Systems Theory; Invariant Manifolds

PACS: 45.10.-b; 95.10.Ce; 96.30.Hf; 96.25.Nc; 45.50.Pk; 45.20.Jj; 05.10.-a; 05.45.-a

\section{INTRODUCTION}

One of the most famous and historically investigated chaotic dynamical systems is the circular restricted problem of three bodies (CR3BP), where the aim is to describe the motion of a point-mass inside the gravitational field generated by two spherical homogeneous bodies moving in circular orbits around their common center of mass. In the last few decades, dynamical systems tools developed for this problem have provided an in-depth understanding of the spacecraft's natural dynamics [1]: the orbits around the Libration points (LPOs) have been computed extensively for the Sun-Earth and Earth-Moon systems, where the approximation of the natural dynamics using the CR3BP is an accurate one [2, 3]-[4, 5, 6], and exploited as energy-efficient trajectories to minimize the fuel consumption of spacecraft for real space missions, such as ISEE-3/ICE (1978), WIND (1994), SOHO (1995) and ACE (1997).

However, future space missions are now targeting deep space celestial bodies, in particular asteroids and planetary satellites. The orbital dynamics around small bodies is considerably complex because many strong perturbations are involved, since they are often far from a spherical shape and their orbits far from circular. More accurate models are required to describe the natural motion and to exploit the LPOs in these systems. The aim of this paper is to extend the model of the CR3BP to a particular example of these types of bodies, that is, the dynamics of a spacecraft in the vicinity of Mars' moon Phobos.

Phobos has been highlighted as a precursor mission to the long term goal of a human mission to Mars [7, 8]: in particular, ESA is scheduling a sample-and-return mission to Phobos $[9,10]$ and NASA is targeting this moon to test a new kind of rover for planetary exploration [11]. The CR3BP, where the two primaries are Mars and Phobos, does not provide a suitable approximation of the natural dynamics in the vicinity of Phobos. This is because Phobos has a highly inhomogeneous gravity field and the eccentricity effect in its proximity is not negligible. In this paper we derive a new model extending the classical CR3BP to an elliptic RTBP (ER3BP) with an inhomogeneous gravity field of the second massive body, described through a spherical harmonics series expansion. 
The natural dynamics of a spacecraft around Phobos are then investigated using numerical continuation $[12,13]$. The procedure used is to identify LPOs in the Mars-Phobos-spacecraft CR3BP and then numerically continue a parameter that incrementally increases the gravity harmonics. Once the corresponding periodic and quasi-periodic LPOs have been identified in this system the orbits are then continued again using the eccentricity as the perturbation parameter. Furthermore, the invariant manifolds of the LPOs in this realistic model of the dynamics are computed, and due to the proximity of the moon, they are applied as natural landing and take-off gateways to and from its surface, identifying the natural trajectories that provide the lowest angle of incidence and escape velocity from their topographical arrival and departure sites.

The outline of this paper is as follows. Section 2 introduces to the reader the analytical and numerical methodologies of dynamical systems theory to identify and compute periodic and quasi-periodic solutions. Section 3 then presents the model of the Mars-Phobos-spacecraft CR3BP and applies these numerical methods to compute the LPOs. Section 4 introduces to the reader the methodology of numerical continuation, that will be used to compute the equivalent LPOs of the classical CR3BP in a more true-life dynamical system that considers the major orbital perturbation in proximity of Phobos. Section 5 presents the Mars-Phobos-spacecraft CR3BP-GH which includes Phobos' gravity harmonics and the LPOs obtained in this system by numerical continuation. Section 6 presents the Mars-Phobos-spacecraft ER3BPGH which includes also the Mars-Phobos orbit eccentricity, and computes the dynamical substitutes of the LPOs for this system. Section 7 concludes the paper and suggests applications for these orbits and their invariant manifolds.

\section{DYNAMICAL SYSTEMS TECHNIQUES FOR THE COMPUTATION OF INVARIANT SOLUTIONS}

This paper investigates the equilibrium, periodic and quasi-periodic solutions of the Mars-Phobos-spacecraft system for use as potential station-keeping orbits in future missions. We begin by highlighting the general definitions and numerical methods of dynamical systems theory (DST) required to identify these invariant motions.

Consider the dynamical system described by the first order differential equations (ODEs):

$$
\left\{\begin{array}{l}
\dot{x}(t)=f(t, x) \\
x\left(t_{0}\right)=x_{0}
\end{array} \Leftrightarrow \phi_{f}\left(t, t_{0}, x_{0}\right): x\left(t_{0}\right) \rightarrow x(t)\right.
$$

where $x$ is the state vector at time $t$, with initial condition $x_{0}$ at $t_{0}, f$ is a general nonlinear (NL) and time-variant (TV) vectorfield, time-continuous and Lipschitz in $x$, and $\phi$ is the flow map solution of the dynamical system, that describes the trajectory $x(t)$ from the initial state. The pointwise linear sensitivity of the trajectory from the initial condition is defined by the state-transition matrix (STM) $\Phi$ of the dynamical system, which is the solution of the variational system of ODEs:

$$
\left\{\begin{array}{l}
\dot{\Phi}_{f}\left(t, t_{0}\right)=A_{f}(t, x) \Phi_{f}\left(t, t_{0}\right) \\
\Phi_{f}\left(t_{0}, t_{0}\right)=I
\end{array}\right.
$$

where $A$ is the Jacobian of the vectorfield evaluated about some reference trajectory.

The trajectory along a finite-time span, as any other function, could be developed in a Fourier synthesis along a continuous frequency spectrum of harmonic functions. This allows classical solutions, such as equilibrium points and periodic orbits, to be characterized by a singular harmonic spectrum at zero or one frequency. Quasi-periodic orbits (QPOs) are their extension to a general discrete spectrum, from one frequency up to potentially infinite terms to reach the general solution synthesis transition from Fourier series to inverse Fourier transform. We can therefore express QPOs as dependent on a discrete number of phases $\varphi$, rather than time, each one with the appropriate harmonic frequency $\omega$ : mathematically speaking, QPO is a n-torus (where $n$ is the dimension of the phase-space). This is expressed by Eq.3, where the torus flow $v$ of a vectorfield $u$ is stated in the phase-space, and the dynamics assume a form known as the invariance equation.

$$
\begin{aligned}
\left\{\begin{array} { l } 
{ \dot { x } ( t ) = f ( t , x ) } \\
{ x _ { Q P O } ( t ) = \phi _ { f } ( t , t _ { 0 } , x _ { 0 , Q P O } ) }
\end{array} \rightarrow \left\{\begin{array}{l}
\frac{\partial x}{\partial \varphi} \omega=u(\varphi, x) \\
x_{Q P O}(\varphi)=v_{u}\left(\varphi, \varphi_{0}, x_{0, Q P O}\right)
\end{array}, \varphi=\varphi_{0}+\omega\left(t-t_{0}\right) \in \mathbb{R}^{n}\right.\right. \\
\quad \mathbf{k} \omega \neq 0, \forall \mathbf{k} \in \mathbb{Z}_{0}^{n}
\end{aligned}
$$

The condition given by Eq.4 represents the non-resonance constraint of the frequency-space, which is just a case that lowers the torus phase-space dimension. 
The propagation of the QPO is usually done by iterated flow mappings, realized by a time-discrete dynamical system: in the phase-space coordinates domain, since their definition is driven by constant frequencies, each phase shift of the flow map is called the rotation number $\rho$.

Finally TV dynamical systems are characterized by forced phases $\tilde{\varphi}$, where their dynamics are constrained: their frequencies $\tilde{\omega}$ correspond to the spectrum of the forcing action. This turns out to be an advantage, because their solutions are explicitly retrievable from integration of the related equations of motion $\dot{\tilde{\varphi}}=\tilde{\omega}$.

\section{Equilibrium solutions of a dynamical system and their computation.}

The first step in our analysis is to identify equilibria from which we can then proceed to find periodic orbits and quasi-periodic orbits. Equilibrium points (EPs) are 0-tori identified as fixed flow roots of the vectorfield, such that

$$
x_{E P}(t)=x_{E P} \Leftrightarrow f\left(t, x_{E P}\right)=0, \forall t
$$

therefore if the vectorfield is time-invariant (TI), EPs are directly the stationary points of the potential function of the system. The computation of a root, $x: f(x)=0$, is performed using Newton's method, which is to assume a linearized approximation of the function in the neighborhood of the sought solution $U_{x}$, and applied iteratively starting from a suitable initial guess $x_{0} \in U_{x}$ for convergence $x_{n} \rightarrow x$.

$$
x_{n+1}=x_{n}-A_{f}^{-1}\left(x_{n}\right) f\left(x_{n}\right)
$$

Once the EPs are identified, their linear stability is described by Lyapunov theory [1], that is, it is determined by the eigenvalues of $A_{f}$ at the EP, which will be asymptotically stable if all of them have real part strictly negative.

\section{Periodic solutions of a dynamical system and their computation.}

A periodic orbit (PO) is a 1-torus defined in Eq.7: $T$ is the associated period, as the minimum time interval within the solution repeats exactly. In addition we define the STM evaluated after one revolution as the monodromy matrix $M$ of the PO.

$$
x_{P O}\left(t_{0}+T\right)=x_{P O}\left(t_{0}\right), \forall t_{0} \rightarrow\left\{\begin{array}{l}
x_{P O}(t)=\phi_{f}\left(t, t_{0}, x_{0, P O}\right) \\
M=\Phi_{f}\left(T+t_{0}, t_{0}\right)
\end{array}\right.
$$

The approach used in DST to compute POs is to reduce the original continuous dynamical system to an appropriate map function of the same state, $P: x \rightarrow P(x)$. In case of TV dynamical systems, the flow map for a propagation time equal to the period of the PO is used, which is called stroboscopic map $P_{T}$. For TI dynamical systems, the approach is due to Poincaré, and consists of the following procedure. First, define a surface of section (or event) of the state-space $\Gamma$, called Poincaré section, which represents a co-1D hypersurface of the state domain (not parallel to the flow) defined by an appropriate constraint $g(x)=0$. Then, define a time-return map $P_{\tau, \Gamma}$ giving the minimum time when the flow, starting from $\Gamma$, returns to the surface itself. Finally, a second map, named the first-return map (Poincaré map) $P_{\phi(\tau), \Gamma}$, associates to the initial state on $\Gamma$ the final state at the first return. Therefore, a PO is identified as the equilibrium fixed point of a first-return map (TI) or stroboscopic map (TV):

$$
\begin{gathered}
x_{0, P O} \in \Gamma \rightarrow P_{\tau, \Gamma}\left(x_{0, P O}\right)=T+t_{0} \rightarrow P_{\phi_{f}(\tau), \Gamma}\left(x_{0, P O}\right)=\phi_{f}\left(P_{\tau, \Gamma}\left(x_{0, P O}\right), t_{0}, x_{0, P O}\right)=x_{0, P O} \\
P_{T}\left(x_{0, P O}\right)=\phi_{f}\left(T+t_{0}, t_{0}, x_{0, P O}\right)=x_{0, P O}
\end{gathered}
$$

Analytical and semi-analytical methodologies, such as the Lindstedt-Poincaré (LP) techniques [1, 14, 15], allow high-order approximations of the NL dynamics to compute periodic and quasi-periodic solutions. They can provide very accurate initial conditions to propagate in the full NL dynamics, but if the POs are highly unstable even very high-orders do not have a very extended region of convergence [1, 14]. In these cases, numerical methodologies must be applied to provide reliable solutions for practical applications, where low-order analytical solutions are used as starting guesses. The numerical technique widely used in DST is the differential corrector (DC) [16]. A DC scheme is essentially a Newton's method used to fine tune the initial condition $x_{0}$ to obtain an accurate orbit that satisfies the objective function $F\left(x_{0}\right)=0$ [1]. DC methods are often called shooting methods, and referred to as single shooting (SS) for the case when only one trajectory is propagated at each iteration, and multiple shooting (MS) when $N$ independent trajectories must be propagated at each iteration of the DC: the technique was initially used for the numerical solution of boundary-value problems [16]. The DC for the computation of a PO of a dynamical system uses its definition as an invariant point of a Poincaré map or stroboscopic map, in a basic SS implementation.

$$
F\left(x_{0}\right)=P_{(\cdot)}\left(x_{0}\right)-x_{0}=0
$$


As a byproduct of the procedure, the propagation time and the STM at the final convergent iteration of the DC will provide $T$ and $M$ of the PO.

Once the POs are computed, their linear stability is described by Floquet theory [1], that is, it is determined by the eigenvalues of $M$ of the PO, which will be stable if all of them have modulus less than 1 . In the case of Hamiltonian systems $M$ is symplectic, therefore its eigenvalues are in couples of complex conjugated, and if the system is also TI the existence of one integral of motion (the energy) means one couple will be 1: this implies that POs are not isolated but are organized in continuous families (parameterized by the energy) developing from an EP with center manifold (EP with a couple of pure imaginary eigenvalues), a result expressed by Lyapunov's center theorem [17].

\section{Quasi-periodic solutions of a dynamical system and their computation.}

This paper focuses on 2-tori, which are quasi-periodic orbits (QPOs). In comparison to POs, defined by a single initial condition on a map, 2D-QPOs are defined by a closed curve of initial conditions $C(\theta)$ (where $\theta$ is the curvilinear coordinate), which is an invariant curve either on a Poincaré (return-map) or stroboscopic map (time-flow).

$$
\begin{gathered}
x_{0, Q P O} \in C(\theta) \in \Gamma \Leftrightarrow P_{\phi_{f}(\tau), \Gamma}\left(x_{0, Q P O}\right)=\phi_{f}\left(P_{\tau, \Gamma}\left(x_{0, Q P O}\right), t_{0}, x_{0, Q P O}\right) \in C(\theta), \forall \theta \in\left[\theta_{m}, \theta_{M}\right): C\left(\theta_{m}\right)=C\left(\theta_{M}\right) \\
x_{0, Q P O} \in C(\theta) \Leftrightarrow P_{T}\left(x_{0, Q P O}\right)=\phi_{f}\left(T+t_{0}, t_{0}, x_{0, Q P O}\right) \in C(\theta), \forall \theta \in\left[\theta_{m}, \theta_{M}\right): C\left(\theta_{m}\right)=C\left(\theta_{M}\right)
\end{gathered}
$$

In particular, as an extension of Lyapunov's center theorem, from every PO with additional center manifold ( $M$ with coupled eigenvalues on the complex unit circle) develops a continuous family of QPOs.

There are a number of numerical techniques used to compute 2D-QPOs, which make use of the description of the invariant curve by truncated Fourier series $C^{F}$ function of the curvilinear coordinate $\theta$ (of period $P$ ),

$$
C^{F}(\mathbf{c}, \theta)=\left[\begin{array}{ccc}
\ddots & \ddots & \\
& & \\
\mathbf{0}_{\mathbf{1 x}\left(\mathbf{2} \mathbf{N}_{\mathbf{C}}+\mathbf{1}\right)} & \mathbf{e}(\boldsymbol{\theta})^{T} & \mathbf{0}_{\mathbf{1} \mathbf{x}\left(\mathbf{2 N}_{\mathbf{C}}+\mathbf{1}\right)} \\
& \ddots & \ddots
\end{array}\right]\left[\begin{array}{c}
\vdots \\
a_{0} \\
\left\{\begin{array}{c}
a_{n} \\
b_{n}
\end{array}\right\}_{n=1}^{N_{C}} \\
\vdots
\end{array}\right]=\mathbf{E}(\boldsymbol{\theta}) \mathbf{c}, \mathbf{e}(\boldsymbol{\theta})=\left[\left\{\begin{array}{c}
\cos \left(\frac{2 \pi}{2} n \theta\right) \\
\left.\sin \left(\frac{2 \pi}{P} n \boldsymbol{\theta}\right)\right\}_{n=1}^{N_{C}}
\end{array}\right]\right.
$$

where the Fourier coefficients $a_{0}, a_{n}, b_{n}$ are retrieved by the discrete Fourier transform and included in the global vector of coefficients c $\left(2 N_{C}+1\right.$ for each coordinate variable). Different DC schemes based on Fourier analysis have been used, see [18, 19, 20]. In this paper the approach of [19] is adopted. Consider a PO and an invariant curve around it on a suitable map, described by the Fourier series $C^{F}$ of Eq.13 function of the transversal parameter $\vartheta$ (a polar anomaly between two state components, that must define the inverse parametrization $\left.\vartheta(x): x=C^{F}(\mathbf{c}, \vartheta), \forall \vartheta\right)$. A DC scheme uses an objective function based on the definition of the invariant curve on a map of Eq.11-12. Since the curve is continuous, it is discretized in $N_{S}$ sample points, by a set of initial parameters $\vartheta_{0}$. Since the method is a LMS technique, the number of Fourier coefficients (for each variable) must be smaller than the number of samplings. The procedure is expressed in a mathematical way in Eq.14, for the general case of a Poincare or stroboscopic map $P_{(\cdot)}$. We have a set of initial conditions $x_{0}$, evaluating the Fourier series at the initial curve parameters. Now MS is performed, finding the set of final conditions of the map. As the parametrization law is invertible, we find the set of parameters function of the final conditions, which are going to be the final curve parameters $\vartheta_{f}$. If the curve is invariant, the same Fourier series evaluated this time at the final curve parameters must correspond to the final conditions; if not, the DC scheme corrects the set of Fourier coefficients.

$$
\left\{\begin{array}{l}
F(\mathbf{c})=P_{(\cdot)}\left(x_{0}\right)-C^{F}\left(\mathbf{c}, \vartheta_{f}\right)=0 \\
x_{0}=C^{F}\left(\mathbf{c}, \vartheta_{0}\right) \\
\vartheta_{f}=\vartheta\left(P_{(\cdot)}\left(x_{0}\right)\right)
\end{array} \quad, \forall \vartheta_{0} \in[0,2 \pi)\right.
$$

Once the invariant curve is identified, it's possible to simulate the $Q P O$ by numerically integrating through iterative mappings, interpolating the intermediate initial condition along the invariant curve.

To perform the stability analysis for QPOs, the method must be modified to provide a 1-1 mapping that determines the invariant curves in a way that resembles the invariance condition of POs. This lies in the fact that we approximate the invariant curve with a truncated Fourier series expansion. The idea is that the set of mappings of the initial conditions along the starting curve constitute a second curve that could also be defined by a related set of final Fourier coefficients $\mathbf{c}_{f}$. This is a map from a set of Fourier coefficients to another (at the same truncated order).

$$
P_{(\cdot), \theta}\left(\mathbf{c}_{0}\right)=\mathbf{c}_{f} \in \mathbb{R}^{6\left(2 N_{C}+1\right)}: C^{F}\left(\mathbf{c}_{f}, \boldsymbol{\theta}\left(P_{(\cdot)}\left(C^{F}\left(\mathbf{c}_{0}, \theta_{s}\right)\right)\right)\right)=P_{(\cdot)}\left(C^{F}\left(\mathbf{c}_{0}, \theta_{s}\right)\right), \forall \theta_{s} \in\left[\theta_{m}, \theta_{M}\right),\left[\left\{\theta_{s}\right\}_{s=1}^{N_{S}}\right]=\theta
$$


If the curve is invariant under the map $P_{(\cdot)}$, the initial Fourier coefficients $\mathbf{c}_{0}$ are a fixed point of the map $P_{(\cdot), \theta}$.

$$
P_{(\cdot)}\left(C^{F}(\mathbf{c}, \theta)\right)=C^{F}(\mathbf{c}, \theta), \forall \theta \Leftrightarrow\left\{\begin{array}{l}
P_{(\cdot), \theta}(\mathbf{c})=\mathbf{c} \\
\Pi=\frac{D}{D \mathbf{c}} P_{(\cdot), \theta}(\mathbf{c})
\end{array}\right.
$$

Going now from sampled points (the set of final conditions) to coefficients, the discrete Fourier transform is used to define the map of Eq.15, which is solved using LMS. The complexity has been transferred to the map itself, because the same LMS fitting is now embedded inside it. Indeed this method requires the computation of the full inverse of a large-scale left pseudo-inverse matrix with dimension $\left(2 N_{C}+1\right)$, requiring far more computational time than the previous one. However it provides, as a byproduct, a monodromy matrix for the stability analysis of the QPO. The related eigenvalues for an invariant curve are organized in circles on the complex plane [21,22], and the Floquet linear stability is therefore given by their magnitude.

\section{Invariant manifolds of a dynamical system and their computation.}

The invariant set of a dynamical system is the domain of initial conditions that remain inside the domain itself for any recursive map composition. The invariant manifold (IM) of an invariant solution is the subset of the state-space that describes the asymptotic trajectories of the solution. There are two kinds of IMs: the linear span of the stable eigenvectors along the orbit and the one of the unstable set, and each of them has two branches, defined by the sign of the eigenvector. For each saddle manifold, to derive the complete tube of each of the four branches, one should integrate the initial conditions provided by the IM along the orbit and derive the set of trajectories. This procedure is known as the globalization of the manifold $[1,23]$.

For the trivial case of the EPs, the IMs are given by the stable and unstable eigenvectors of $A_{f}$, while for POs an analogous approach is related to $M$. For QPOs, the computation of the related IMs depends on the method used to compute the orbit. Using semi-analytical techniques provides directly an expression of the trajectory: the QPO is approximated by successive mappings, so the STM at each subinterval is used in place of $M$. Using the numerical method of Eq.16, the byproduct is a global monodromy matrix $\Pi$, that gives directly the IMs of the full invariant object. The numerical solution of the eigenvalue problem for the large-scale matrix $\Pi$ produces spurious eigenvalues: the approach to select the eigenvector with least computational inaccuracy for each manifold is described in [21, 22].

\section{THE MARS-PHOBOS CR3BP}

The study of the Mars-Phobos-spacecraft system is conducted in the first instance with the CR3BP model [1], which is to consider the gravitational effect of the spacecraft negligible with respect to the two massive bodies (1: Mars; 2 : Phobos), and to choose a rotating reference frame centered in the system's barycenter, where the first axis is aligned along the two bodies and the third one along the out-of-plane direction of their orbital plane. The mathematical approach of the 3BP is conducted with adimensional units, choosing as unit of mass the total mass of the system, and as time and unit of length $L$, respectively the mean anomaly and the semi-major axis of the orbit of the two bodies. The only parameter of the normalized TI CR3BP is the mass factor $\mu$, the normalized mass of the second body, which for the case of Mars-Phobos couple is $\mu=1.66059511088139 \cdot 10^{-8}$. Note that the mass parameter of the system is very small, if compared to other cases studied so far in the Solar System. Second of all, the length unit of the system is very small too since the altitude of Phobos' orbit is less than twice the radius $R_{1}$ of Mars $\left(R_{1} / L=36 \%\right)$, an unusual condition for the pairs of primaries of the Solar System. The equations of motion (EoM) of the CR3BP are stated in Eq.17, where $\mathbf{q}$ is the position of the spacecraft, $\mathbf{x}$ is the resulting state of the vectorfield $\mathbf{f}$ of the dynamical system reduced to the first order, $u(\mathbf{q})=u_{G_{1}}(\mathbf{q})+u_{G_{2}}(\mathbf{q})$ is the gravitational potential of the two massive bodies (only function of $\mathbf{q}$ ), the notation $\odot / *$ denotes the partial derivative of the function $\odot$ with respect to the variable $*$, and since the frame angular velocity is $\omega=[0 ; 0 ; 1]$ the notation is simplified by defining the skew-symmetric matrix $\mathbf{W} \in \mathbb{R}^{3 \times 3}: \mathbf{W} \cdot(\cdot)=\omega \wedge(\cdot)$ and the related matrix $\mathbf{P}=\mathbf{W}^{2}$.

$$
\mathbf{x}=\left[\begin{array}{c}
\mathbf{q} \\
\dot{\mathbf{q}}
\end{array}\right], \dot{\mathbf{x}}=\mathbf{f}_{\mathbf{3 B}}(\mathbf{x})=\mathbf{A} \mathbf{x}+\left[\begin{array}{c}
\mathbf{0}_{\mathbf{3} \mathbf{1}} \\
u_{/ \mathbf{q}}(\mathbf{q})
\end{array}\right], \mathbf{A}=\left[\begin{array}{cc}
\mathbf{0}_{\mathbf{3}} & \mathbf{I}_{\mathbf{3}} \\
-\mathbf{P} & -2 \mathbf{W}
\end{array}\right]
$$

The Hill's sphere of influence is the part of the position-space around each body where the dynamics is dominated by its own gravity field, and its radius for Phobos is $0.17 \%$ of the distance from Mars, which is a mean altitude of only 5.5km: the "physical boundary" within orbit around Phobos at low energies is very close to the body, 

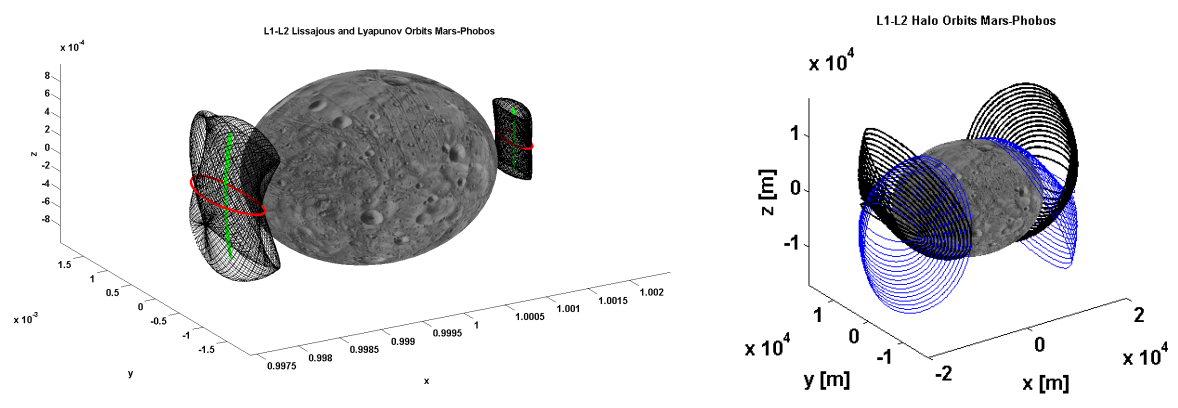

FIGURE 1. LPOs in the Mars-Phobos CR3BP. On the left, example of two planar (red) and vertical (green) Lyapunov orbits around each LP and one Lissajous (black) orbit of high width around them. On the right, families of Northern (black) and Southern (blue) Halo orbits. Mean ellipsoid for Phobos surface.

and considering the fact that Phobos is very irregular in its shape the related altitude is of only $3.5 \mathrm{~km}$. Therefore it's impossible to naturally orbit around Phobos with a Keplerian motion. It's important to point out that this collapsing effect of the realm of attraction of the secondary towards its surface is a result of the two peculiarities of the system. In particular only five other couples in the Solar System can be characterized by the same effect.

EPs. The stationary points of the effective potential are the five EPs of the $3 B$ dynamics in the rotating frame [1]: the three collinear libration points (LPs), aligned with the two bodies (two close to the secondary, $L_{1}$ in inferior and $L_{2}$ in superior conjunction, and $L_{3}$ in opposition), and the two equilateral $L_{4}$ and $L_{5}$, equidistant from them in the two quadrature configurations. The LPs are computed as explained in section 2. In particular, the collinear couple $L_{1-2}$ is very important in mission design, as their distance from the secondary formally defines its sphere of influence. The collinear LPs are located at an altitude of only $3.5 \mathrm{~km}$ from Phobos. Regarding the stability properties, $L_{1-2}$ are characterized by a vertical center manifold, and on the orbital plane by a center $\times$ saddle behavior, which is unstable. This means that the region around these LPs is characterized by two manifolds of POs, their IMs, and transit (inside the IMs) and non-transit (outside the IMs) orbits between the realms of attraction of the two bodies [1].

POs. Around the collinear LPs, two 1-parameter families of POs exist at increasing size/energy from them, called planar and vertical Lyapunov orbits [1]. As their energy increases, they encounter bifurcations: the first one of the planar orbits gives birth to the two important symmetrical branches of Northern and Southern Halo orbits [2, 3]. The families of Lyapunov orbits are here computed using the LP method. The method approximates the NL terms of the gravity potential as a series expansion of Legendre polynomials, and computes the coefficients of the series expansion of only the periodic normal modes of these approximated dynamics [1,14]. The two families of Halo orbits are computed through the numerical DC introduced in Section $2[18,3]$. The Poincaré map is taken at $g(\mathbf{x})=y$, and due to the symmetries of the CR3BP, the objective function is to erase the final velocities $\dot{x}$ and $\dot{z}$ correcting the initial state $x$ and $\dot{y}$. The initial guess for the DC is given by the Richardson's solution [24], which is a LP expansion at order 3. These four families of POs have been computed up to the intersection with Phobos, and they are all unstable. Fig.1 shows some example of POs in the Mars-Phobos CR3BP.

QPOs. The collinear region is finally characterized by a family of 2D-QPOs at increasing size around each PO with central part, and iso-energetic with it. Due to the pattern of the projections on the coordinate planes, they are widely know as Lissajous orbits [1]. We computed the manifold of the 2-parameter family of Lissajous orbits around the Lyapunov orbits directly with the LP expansion of 2-tori [1, 14]. Fig.1 shows some example of QPOs in the Mars-Phobos CR3BP. Related IMs are then computed as explained in section 2.

In conclusion, an orbital perturbation analysis, conducted in the framework of the relative motion in proximity of Phobos, shows that the complete inhomogeneous gravity field and the orbital eccentricity of Phobos are the main orbital perturbations that affect the dynamics in proximity of the moon. 


\section{THE NUMERICAL CONTINUATION TECHNIQUE}

From the analysis of the perturbations around Phobos, the CR3BP is not a suitable model for the orbital dynamics in proximity of the moon. Due to the highly unstable behavior, the families of LPOs are therefore not reliable for practical applications, as tracking these solutions will require a high station-keeping cost. In section 5 and 6 , the dynamical substitutes of the LPOs are derived in a more realistic model that considers the major orbital perturbations in proximity of Phobos, which we previously pointed out are its highly inhomogeneous gravity field and its orbital eccentricity. The computation of the LPOs in this system will be undertaken numerically, by the use of the numerical continuation (NC) technique.

The basic idea of the NC method is given by considering two slightly different functions $F(x)$ and $G(x)$ on a set $U_{x}$,

$$
x \in U_{x}: F(x) \approx G(x) \Rightarrow\left\{\begin{array} { l } 
{ H ( x , \sigma ) = ( 1 - \sigma ) F ( x ) + \sigma G ( x ) = H ( y ) } \\
{ y = [ \begin{array} { l } 
{ x } \\
{ \sigma }
\end{array} ] }
\end{array} \leftrightarrow \left\{\begin{array}{l}
H(x, 0)=F(x) \\
H(x, 1)=G(x)
\end{array}\right.\right.
$$

such that their linear combination produces a global function $H$, which depends on $0 \leq \sigma \leq 1$ (named the continuation parameter). Suppose that $F$ has a known root. A Newton's method could use it as a first guess to numerically compute the root of $H$ for $\sigma$ small. Therefore intuitively, if the level of perturbation in the final function $G$ where we want to find the solution (we call it the substitute of the original root) is too high, we can just increment the parameter slowly, solving Newton's methods by iteratively using as an initial guess the solution found at the previous step.

In this paper the pseudo-arclength $\mathrm{NC}[12,13]$ will be implemented in different DC schemes, using a predictor for the initial guess along the continuation curve, given either by the kernel of the linearized $H$ or by its first-order finite differences, and an adaptive step strategy, based on the control of the number of DC iterations.

\section{THE MARS-PHOBOS CR3BP-GH}

The modeling of the complete gravity field of convex bodies is provided by a spherical harmonics series expansion, known as gravity harmonics (GHs). From a previous paper [25] that collects the data obtained through Viking observations, we are provided with a model of the Phobos gravity field. The related GHs arrive until degree and order 4. The key point is that not only the revolution of Phobos around Mars and the rotation around its spin axis are synchronous, but they are also respectively equatorial and zero-tilted [26], therefore Phobos' attitude is approximately fixed in the rotating frame of the CR3BP, and the EoM with GHs (named CR3BP-GH) remain autonomous. In particular, the error assuming a fully TI CR3BP-GH for the Mars-Phobos case oscillates between a minimum of $0.30^{\circ}$ and a maximum of $1.90^{\circ}$, and the dynamics of this misalignment is much slower than the timescale of a mission segment around Phobos (period of 2.26 terrestrial years).

The gravitational potential $u_{G}$ is described by the series expansion of spherical harmonics [27], in a Planet-Centered Planet-Fixed frame (PCPF), with spherical coordinates (TSE frame: position described by magnitude $r$, co-latitude $\vartheta$, East-longitude $\psi$ with respect to a Prime Meridian), where $G M$ and $R$ are the gravity parameter and equivalent-volume radius of the body.

$$
\left\{\begin{array}{l}
u_{G}(\mathbf{q})=u_{G}(r, \vartheta, \psi)=\frac{G M}{R} \sum_{n=0}^{\infty}\left(\frac{R}{r}\right)^{n+1} \sum_{m=0}^{n} C_{n}^{m}(\psi) P_{n}^{m}(\cos \vartheta) \\
C_{n}^{m}(\psi)=C_{n, m} \cos m \psi+S_{n, m} \sin m \psi=J_{n, m} \cos m\left(\psi-\lambda_{n, m}\right)
\end{array}\right.
$$

Double expansion is conducted with degree $n$ and order $m$, using the Legendre associated polynomials $P_{n}^{m}$, function of the co-latitude, which are scaled with two $n, m$-dependent coefficients linearly combined with harmonic functions of the longitude: $C$ and $S$ (or related magnitude $J$ and phase $\lambda$ ) are the Stokes coefficients pair for each degree and order of the series expansion, so they are the "physical measures input" needed for the computation. A similar series expansion is available for describing the real shape of Phobos [28]: for consistency, the related shape harmonics (SHs) will so be used from now on to model the surface of Phobos for practical applications. Now from the definition of the potential, the gravitational acceleration of Phobos is obtained through the spherical gradient $\nabla_{T S E}$, then rotated in the PCPF frame. For the application of the DC schemes and the stability analysis, we need also the hessian matrix of the potential which is computed from the hessian definition for a general curvilinear coordinates basis, that makes use of the metric matrix $\mathbf{g}$ and the Christoffels' symbols $\Gamma$ of the coordinate transformation [29].

The gravitational potential of Phobos is extended in the GHs series expansion: $J_{0,0}=1$ is the basic spherical term used in the CR3BP, degree-1 terms are null for barycentric frames; therefore, the net gravitational perturbation of Phobos 

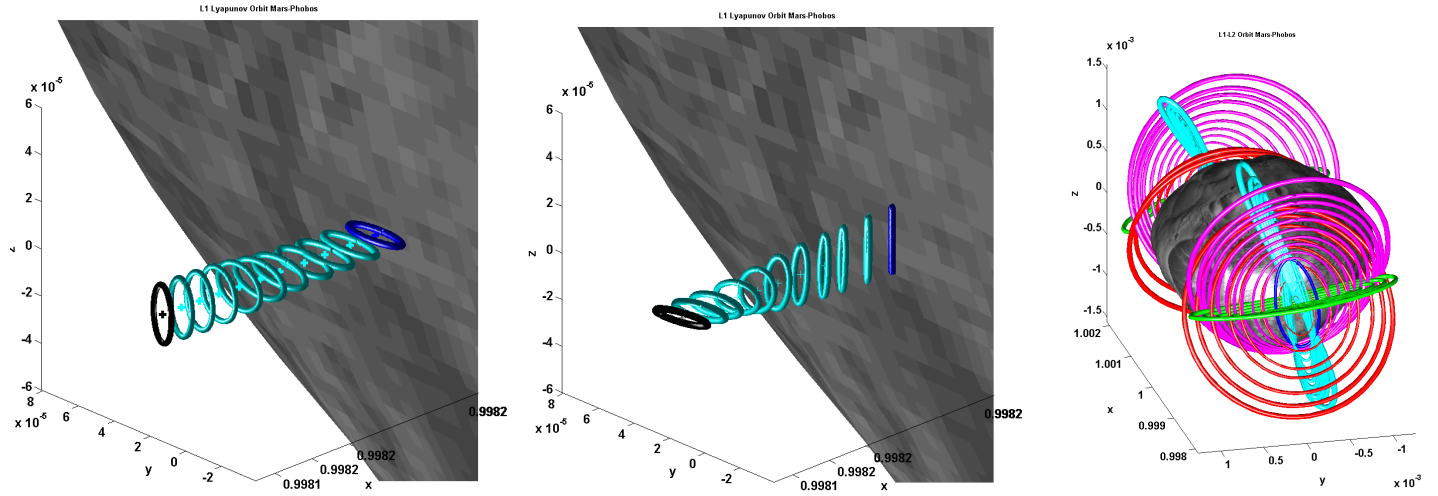

FIGURE 2. POs in the Mars-Phobos CR3BP-GH. First two figures show the NC with respect to the GHs of a small-energy planar and vertical Lyapunov orbit around $L_{1}$ of the CR3BP (blue) to the dynamical substitute in the CR3BP-GH (black), together with the current LP. Last figure shows the families A (red), B (green), C (magenta), D (cyan), and ancestor orbit (blue) of the family CD. Shape harmonics series expansion for Phobos surface.

$\mathbf{a}_{\mathbf{G}}^{\mathbf{p}}$, starting from $J_{2,0}=0.1051$, is added to the CR3BP vectorfield to derive the EoM of the CR3BP-GH,

$$
\begin{gathered}
\dot{\mathbf{x}}=\mathbf{f}_{\mathbf{3 B}}(\mathbf{x})+\mathbf{f}_{\mathbf{G H}}(\mathbf{x})=\mathbf{A} \mathbf{x}+\left[\begin{array}{c}
\mathbf{0}_{\mathbf{3 x} \mathbf{1}} \\
u_{G_{1} / \mathbf{q}}(\mathbf{q})+u_{G_{2} / \mathbf{q}}(\mathbf{q})
\end{array}\right]=\mathbf{f}_{\mathbf{3 B}}(\mathbf{x})+\left[\begin{array}{c}
\mathbf{0}_{\mathbf{3 x} \mathbf{1}} \\
\mathbf{a}_{\mathbf{G}_{\mathbf{2}}}(\mathbf{q})
\end{array}\right] \\
\mathbf{a}_{\mathbf{G}_{\mathbf{2}}}^{\mathbf{p}}(\mathbf{q})=\mathbf{A}_{3 B F}^{P_{2} C P_{2} F}\left(\mathbf{A}_{T S E}^{P_{2} C P_{2} F}\right)^{T} \nabla_{T S E} u_{G_{2}}\left(\mathbf{A}_{T S E}^{P_{2} C P_{2} F}\left(\mathbf{A}_{3 B F}^{P_{2} C P_{2} F}\right)^{T}\left(\mathbf{q}-\left[\begin{array}{c}
1-\mu \\
0 \\
0
\end{array}\right]\right)\right)
\end{gathered}
$$

where two attitude matrices A are used: the one between the 3B frame and the Phobos PCPF one is TI and constant due to the physical and orbital peculiarities of Phobos as explained previously, and the one between the TSE frame and the PCPF one depends on the spherical coordinates $\vartheta$ and $\psi$.

EPs. The EPs of the CR3BP-GH are calculated as usual, with the potential function now considering the complete gravity field of Phobos through Eq.19-20. The outcomes obtained show that the displacement of $L_{1}$ and $L_{2}$ from the values of the basic model is quite relevant, in terms of altitude reaching the $\mathbf{2 0 \%}$, which shows how inaccurate this model is for our practical applications. The stability properties of the manifold are instead the same. Also, it must be reported that the truncated GHs model mathematically provides additional EPs to the system, but they are all inside the Phobos figure and so they are not directly exploitable.

POs. To derive the substitutes of the periodic LPOs in the Mars-Phobos C3BP-GH, NC is used starting from the initial conditions of the families of POs computed in the basic CR3BP. The DC scheme step looks for invariant points of the Poincaré map through Eq.10. The continuation parameter $\sigma$ is, intuitively, a scaling factor, from 0 to 1, to weight the GHs acceleration in the vectorfield.

$$
\dot{\mathbf{x}}=\mathbf{h}\left(t, \mathbf{x}, \sigma_{G H}\right)=\mathbf{f}_{\mathbf{3 B}}(\mathbf{x})+\sigma_{G H} \mathbf{f}_{\mathbf{G H}}(\mathbf{x}) \rightarrow \phi_{\mathbf{h}}\left(t, \mathbf{x}, \sigma_{G H}\right)
$$

Note that the problem is still conservative and so an appropriate Jacobi integral of the augmented vectorfield is available: therefore, POs are continued in a differential way with respect to the state of the current LP, using a variant Poincaré map $g\left(\mathbf{x}, \sigma_{G H}\right)=y-y_{E P}\left(\sigma_{G H}\right)$, and with a fixed-energy constraint with respect to the energy of the LP. Therefore, the NC of the POs embeds also the computation of the current LP: this choice of a differential NC has been proved to be faster than continuing the absolutes state, since it requires less DC iterations on equal step size.

The NC is performed for a tenth of POs for each of the four families of the CR3BP, for both LPs. Fig.2 shows what physically happens, as the small planar and vertical Lyapunov orbits follow the current LP and they are distorted, mostly in the second half of the continuation. In particular, the vertical Lyapunov orbit deploys itself in an elliptical orbit too, as a sign of strong 3D-coupled dynamics, and counterintuitively, substitutes of planar orbits are mostly vertical and substitutes of vertical ones are mostly planar. At the end, visually four families of POs in the CR3BPGH are identified, no more symmetric and highly distorted from the POs of the classical CR3BP. They have been 

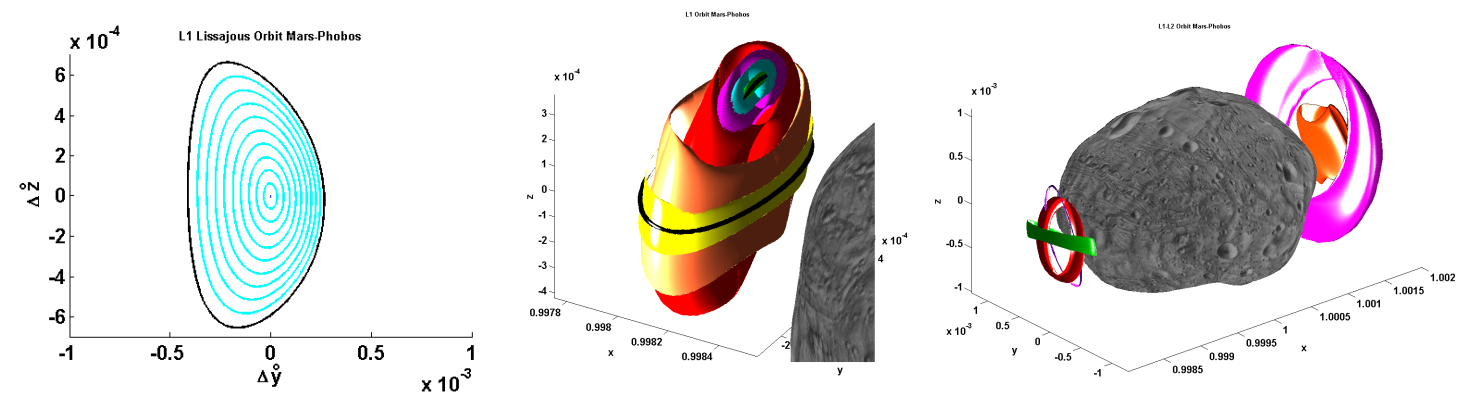

FIGURE 3. QPOs in the Mars-Phobos CR3BP-GH. On the left, family of invariant curves around one medium-energy PO of the family A of $L_{1}$. In the center, example of the family of QPOs around two iso-energetic medium-energy POs (black) of the families A and B of $L_{1}$. On the right, example of QPOs of different energy and width around the LPs: three medium-energy QPOs of family $\mathrm{AB}$ (red and green with small-width, orange with high-width) and two high-width QPOs of family $\mathrm{C}$ (around the ancestor orbit and at high-energy). Shape harmonics series expansion for Phobos surface.

named families A, B, C, and D. To refine the families we then take one of the POs just computed for each of them and perform a NC with respect to the differential Jacobi integral used as a fixed constraint previously, in the final CR3BP-GH for $\sigma_{G H}=1$. The final outcomes are represented in Fig.2 for both $L_{1}$ and $L_{2}$. Larger orbits have shape similar to the four families of POs of the CR3BP, but they are clearly tilted. The manifolds of these LPOs are the classical saddle $\times$ center for the A, B, and C families, while the $D$ family does not have a central part at all. With this $\mathrm{NC}$, we found that the families $\mathbf{C}$ and $\mathbf{D}$ are actually two branches of the same global family (CD), since they are connected through an ancestor orbit at the lowest energy. In particular the reverse NC of this ancestor orbit back to the CR3BP produces a closed loop continuation curve, without a dynamical substitute in the basic problem.

QPOs. To compute the families of 2-tori around each $\mathrm{PO}$ of the families A, B, and C of the CR3BP-GH, and the related IMs, the numerical methodologies based on Fourier analysis explained in Section 2 are used, using a Poincaré map. There are two additional constraints: the iso-energetic requirement of the points along the invariant curve with the backbone PO; and then, since this is indeed a family, to compute only one QPO at each step the DC is embedded in a NC with respect to the width of the invariant curve [19], to provide finally the two families $\mathrm{AB}$ and $\mathrm{C}$ of QPOs around each LP (families A and B of tori are connected each other) highlighted in Fig.3.

\section{THE MARS-PHOBOS ER3BP-GH}

Despite the fact that the orbital eccentricity $e=0.0156$ of Phobos is not particularly high, the perturbation has a significant effect on the LPOs, as an indirect effect of the long-cited collapse of the collinear region towards the moon. The addition of the eccentricity in the CR3BP has already been investigated in the past [30,31], and it has been extensively studied in the same barycentric and rotating frame of the circular case (now with a forced law of motion for $\omega$ and $\dot{\omega}$ ), with a time-dependent length unit corresponding to the distance between the two massive bodies (called pulsating frame), and a non-uniform time variable corresponding to the true anomaly $v$. In our case, since we are focused in a closed relative motion perspective around Phobos, and also we need to consider the GHs of the moon, the classical pulsating frame of the ER3BP is likely to be avoided, because it produces different time-dependent scalings for each GHs at different degree. The only possibility to overcome this situation, is to consider a reference frame, still rotating with the elliptic motion, but centered in Phobos and with a fixed length unit (semi-major axis). Regarding the time variable, since the vectorfield is used not only for integration but also in NC schemes, the simpler choice is to consider a fixed time unit too, and use the mean anomaly: this because the sensitivity of the flow with respect of $v_{0}$ could be be retrieved appending $v$ in the state vector $\mathbf{x}=[\mathbf{q} ; \dot{\mathbf{q}} ; \boldsymbol{v}]$. The EoM of our ER3BP-GH are:

$$
\dot{\mathbf{x}}=\left[\begin{array}{ccc}
\mathbf{0}_{\mathbf{3}} & \mathbf{I}_{\mathbf{3}} & \mathbf{0}_{\mathbf{3} \mathbf{1}} \\
-\frac{(1+\cos v)^{4}}{\left(1-e^{2}\right)^{3}} \mathbf{P}+2 \frac{(1+e \cos v)^{3} e \sin v}{\left(1-e^{2}\right)^{3}} \mathbf{W} & -2 \frac{(1+e \cos v)^{2}}{\left(1-e^{2}\right)^{3 / 2}} \mathbf{W} & \mathbf{0}_{\mathbf{3} \mathbf{1}} \\
\mathbf{0}_{\mathbf{1 \times 3}} & \mathbf{0}_{\mathbf{1 \times 3}} & 0
\end{array}\right] \mathbf{x}+\left[\begin{array}{c}
\mathbf{0}_{\mathbf{3} \mathbf{1} \mathbf{1}} \\
\frac{(1+\cos v)^{2}}{\left(1-e^{2}\right)^{3 / 2}}
\end{array}\right]
$$



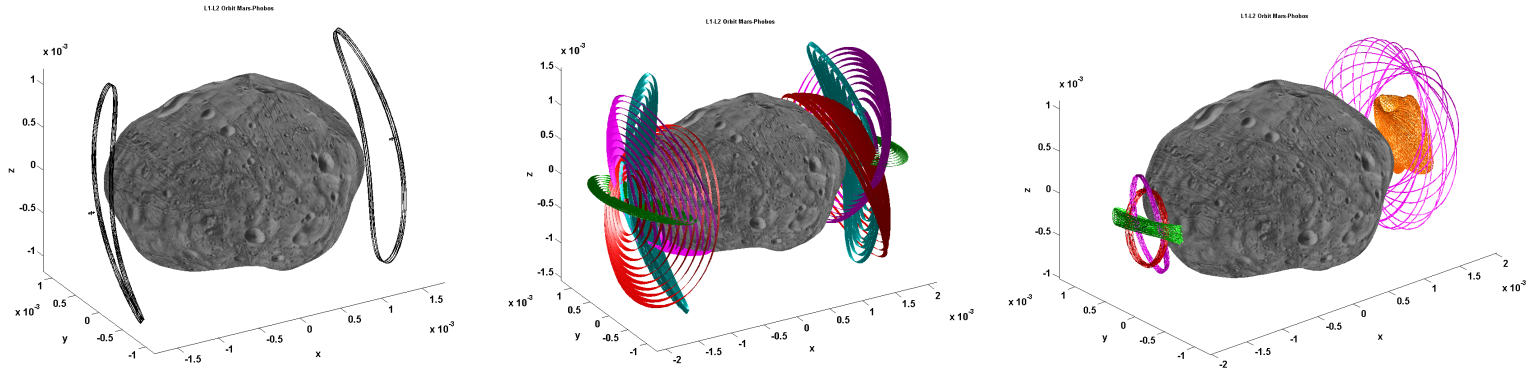

FIGURE 4. LPOs in the Mars-Phobos ER3BP-GH. On the left, the two iso-periodic families of POs around the oscillating LPs. In the center, the families of 2-tori: A (red), B (green), C (magenta), D (cyan). On the right, example of 3-tori of different size and width around the LPs: three medium-size QPOs of family AB (red and green with small-width, orange with high-width) and two high-width QPOs of family C (around the ancestor orbit and at high-size). Shape harmonics series expansion for Phobos surface.

Summarizing the eccentricity produces a time-dependent perturbation to the dynamics of the circular case. This means that the solutions of the elliptic dynamics in the phase-space have one phase constrained to follow the dynamics of $v$. The NC of the invariant solutions computed in the CR3BP-GH will increment by one their phase-space and produce dynamical substitutes in the ER3BP-GH that oscillate around the original ones.

POs. Since the dynamics of the ER3BP-GH is TV, and the eigenvalues of the LPs of the CR3BP-GH are different from the orbital frequency of Phobos, no physical EPs exist in this non-pulsating system. The LPs now become $P O s$, as they oscillate along the LPs of the circular case with an amplitude about $260 \mathrm{~m}$, which is quite relevant at the length scale of Phobos for practical applications.

Apart from the dynamical substitutes of the ancestor EPs, isolated POs in the ER3BP-GH develop when the original $P O$ in the circular case is resonant with $v$. In this case, the characteristic curves of the period of the POs for the four families of the CR3BP-GH show that the two families D have one PO with period $\pi$, which is the usual overresonance 2:1 (resonances with high denominators are not considered). The dynamical substitutes of these two synchronous POs in the ER3BP-GH are still POs, with period $2 \pi$ : this gives rise to two iso-periodic families of POs in the elliptic problem (see Fig.4), since the circular problem is a singular case for the definition of $v_{0}$. The computation of these POs could be undertaken with the DC used previously for POs but using a $2 \pi$-flow map, and embedded in a NC where the parameter is the eccentricity [32].

$2 D$-QPOs. These orbits are the dynamical substitutes of the POs of the CR3BP-GH in the general non-resonant case. They are computed with the DC used previously to compute their invariant curve, but with some important differences. First, the invariant curves are now defined on a stroboscopic map, with the period fixed to the one of the original PO. Second, the invariant curve parameter is fixed to be $v$ on the stroboscopic map, so the related rotation number is directly retrievable by integration of the EoM. The only constraint to add regards the longitudinal phase indeterminancy of the torus [18]. The DC is embedded in a $\mathrm{NC}$ with respect to the eccentricity, to find the invariant curve in the ER3BP-GH from an original PO [20]. Once one QPO is obtained, the refinement for the related family can be achieved changing the continuation parameter to be the stroboscopic time, at the eccentricity of Phobos. Fig. 4 shows the A, B, and CD families of QPOs in the Mars-Phobos ER3BP-GH.

In particular, an unexpected coupling between GHs and eccentricity has been found. As a difference from the basic $C R 3 B P$, the characteristic curve of one family of POs at low-energy has a local maximum: close to this region of the families A, two POs with same period have the same invariant curve for a certain eccentricity, so all the original POs in the CR3BP-GH with period larger than the one of this couple do not have a 1-1 dynamical substitute QPO in the elliptic case. This is highlighted in Fig.5

This algorithm for QPOs naturally embeds also the resonant cases, as their invariant curve on a multiple-return stroboscopic map has a flat null rotation number profile. Therefore this method is used to continue the synchronous $P O$ of the D families, and it is clearly superior to the dedicated one for POs, because it provides not only one initial condition in the ER3BP-GH, but directly a curve of invariant points that describes the iso-periodic family of POs. 


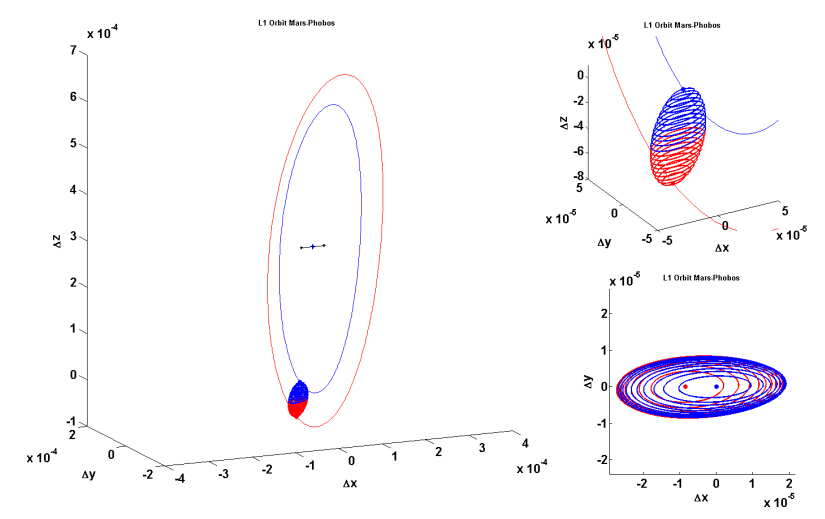

FIGURE 5. LPOs in the Mars-Phobos ER3BP-GH. Invariant curves of the stroboscopic map obtained by NC with respect to the eccentricity from CR3BP-GH to ER3BP-GH of a small-energy PO (blue) of the A family around $L_{1}$, showing that this orbit does not have a dynamical substitute QPO in the ER3BP-GH at the eccentricity of Phobos. This continuation actually links the starting PO to another one (red, of same family and period) in the globalized ER3BP-GH $(t, v, \mathbf{x}, e)$.

3D-QPOs. These QPOs with three frequencies are the dynamical substitutes of the 2-tori of the CR3BP-GH, and each of them is identified by an invariant 2-torus. They have been computed using the DC scheme, embedded in a $\mathrm{NC}$ with respect to the eccentricity, of the classical MS method $[33,16]$ that satisfies the continuity between the subintervals where the QPO trajectory is sampled. The resulting linear system of the DC is in this case underdetermined. In conclusion, Fig.4 showcases some samples simulated of the AB and $\mathbf{C}$ families of 3-tori around $L_{1}$ and $L_{2}$ of the ER3BP-GH. An example of the related IMs is shown in Fig.6.

\section{CONCLUSION}

The analysis of the natural dynamics inside Phobos' realm of attraction undertaken in this paper has revealed some potential mission opportunities around the libration points $L_{1}$ and $L_{2}$. First the classical families of periodic and quasi-periodic LPOs in the basic CR3BP have been computed for the Mars-Phobos system using the well-known methodologies developed in literature. The LPOs are well-known exploitable solutions for orbiting about the smaller primary and they provide energy-efficient trajectories for interplanetary transfers of spacecraft through their IMs: unfortunately they are highly unstable and station-keeping is required. For the Mars-Phobos system, the altitude of the LPs moves very close to Phobos irregular surface: in this situation, the basic dynamical approximation provided by the CR3BP falls short, and in particular two differential orbital perturbations become relevant in describing the natural relative motion around this moon, which are its complete highly inhomogeneous gravity field, and its orbital eccentricity.

The dynamical substitutes of the LPOs have been computed in an ER3BP-GH, through NC techniques: in particular, whereas the time-dependent eccentricity effect has already been studied throughout the years, no deep analysis has been undertaken to consider the additional GHs of the secondary body; the Mars-Phobos-spacecraft system is particulary suitable for two reasons: firstly because their perturbation is significant due to the collapse of the collinear region, and then because the additional physical and orbital characteristics make this system so unique to preserve the time-invariance of the hamiltonian model. In particular, the effect of the GHs produces families of POs and QPOs highly distorted from the classical case, whereas the effect of the eccentricity is to make the real motion to oscillate around these solutions with a considerable amplitude for the proximity of Phobos.

The resulting LPOs of this improved upgraded dynamical model considerably lower the station-keeping demand exploiting the natural dynamics of the system: around each collinear side of Phobos, they are constituted by a 1parameter family of iso-periodic POs vertically developed with the period of Phobos revolution around Mars, three 1-parameter families of 2-tori QPOs, and two 2-parameter families of 3-tori QPOs, all of them very close to the surface of the moon and highly unstable. Since the orbits are close to Phobos, no homoclinic nor heteroclinic connections are available to naturally move around this Martian moon, but the IMs of these LPOs could be exploited as natural landing or take-off gateways to and from the surface of Phobos. In particular, the inside branch of the IMs have been computed and a related performance analysis has shown that high-efficient natural tangential landing paths 

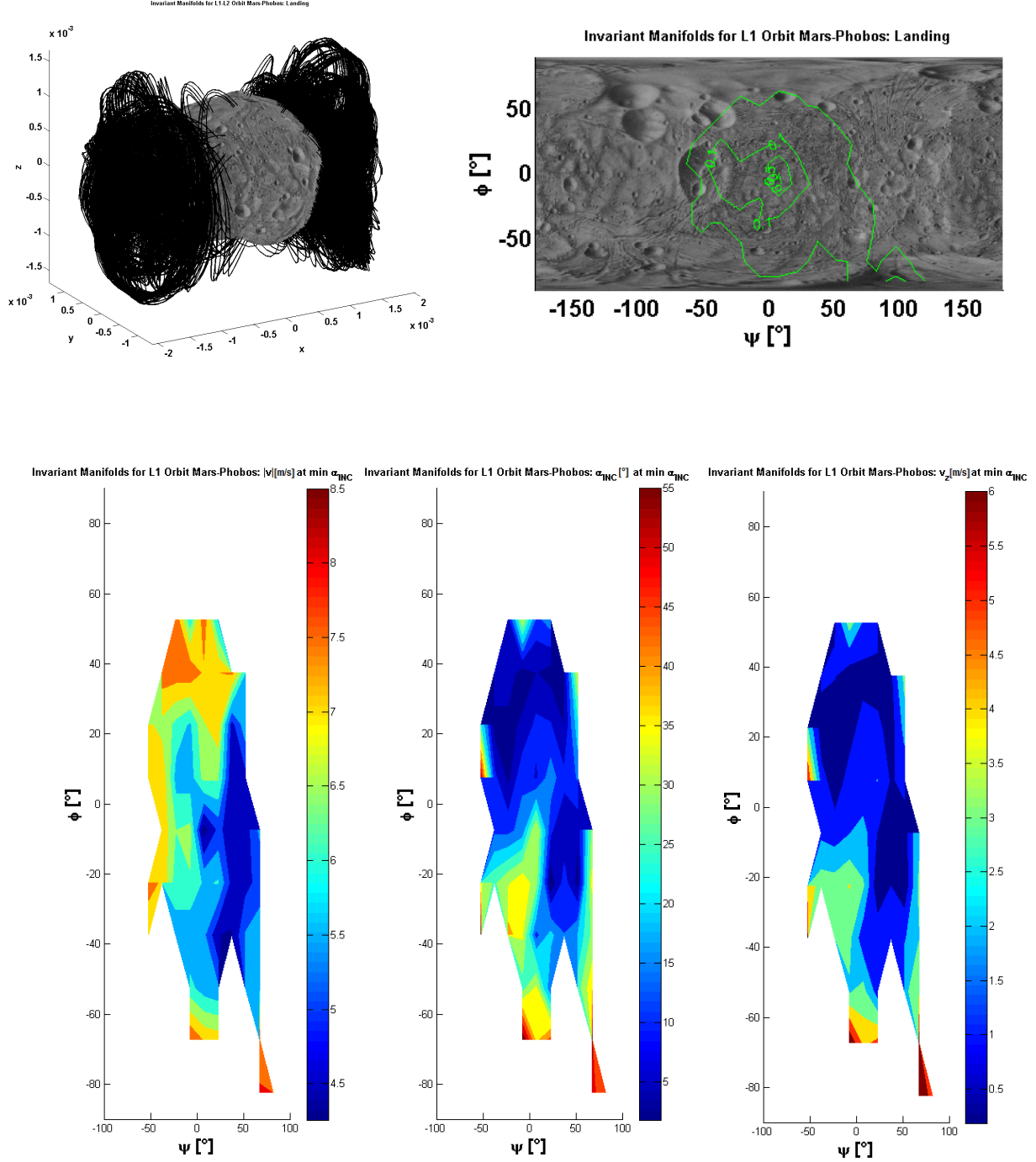

FIGURE 6. IMs in the Mars-Phobos ER3BP-GH. On the top-left, inside branch of the tube of unstable IMs from the families of 3-tori LPOs. On the top-right, region of possible landing sites of the family $\mathrm{AB}$ of $L_{1}$ on Phobos surface modeled through shape harmonics series expansion: inner lines show subregions where the $10 \%, 50 \%, 90 \%$ levels of the cumulative distribution of the IM simulated landed (same number of trajectories from each departing orbit simulated). On the bottom, related performances of the trajectories that provide the min incidence at the touch-down, as a function of the longitude and latitude of the landing site: landing velocity modulus, angle of incidence, downward vertical velocity.

and low escape velocity injections (far less than the 2B $\Delta \mathrm{v}$ value) are available for a fair region of topographical collinear-faced sites on Phobos: these trajectories have the potential to be exploited for future sample-and-return missions to this moon.

\section{ACKNOWLEDGMENTS}

This work has been supported by the European Commission through the Marie Curie fellowship PITN-GA-2011289240 "AstroNet-II". We are thankful to Prof. J.J. Masdemont and Prof. G. Gómez (IEEC) for the assistance provided in the computation of LPOs in the Mars-Phobos CR3BP through high-order LP series expansions in the early stage of this research. 


\section{REFERENCES}

1. W.S. Koon, M.W. Lo, J.E. Marsden, S.D. Ross. Dynamical Systems, the Three-Body Problem and Space Mission Design. New York City: Springer-Verlag, 2011.

2. R.W. Farquhar. The Control and Use of Libration-Point Satellite. Ph.D. Thesis. Stanford University, 1968.

3. K.C. Howell. Three-Dimensional, Periodic, 'Halo' Orbits. Celestial Mechanics, 32(53), pp. 53-71 (1984).

4. C.C. Conley. Low Energy Transit Orbits in the Restricted Three-Body Problem. SIAM Journal of Applied Mathematics, 16, pp. 732-746 (1969).

5. R. McGehee. Some Homoclinic Orbits for the Restricted Three-Body Problem. Ph.D. Thesis. University of Wisconsin, 1969.

6. W.S. Koon, M.W. Lo, J.E. Marsden, S.D. Ross. Heteroclinic connections between Periodic Orbits and Resonance Transitions in Celestial Mechanics. Chaos, 10, pp. 427-469 (2000).

7. J.B. Hopkins, W.D. Pratt. Comparison of Deimos and Phobos as Destinations for Human Exploration, and Identification of Preferred Landing Sites. AIAA Space 2011 Conference \& Exposition, Long Beach, 2011 September 27-29.

8. P. Lee. Phobos and Deimos Update. 7th SBAG Meeting, Pasadena, 2012 July 10-11.

9. M. McCaughrean. ESA's Report to the 39th COSPAR Meeting. Noordwijck: ESA Communications, 2012.

10. D. Koschny. Phootprint: An ESA Mission Study. Mars Concepts and Approaches Workshop, Houston, 2012 June 12-14.

11. M. Pandika. Stanford researchers develop acrobatic space rovers to explore moons and asteroids. [Online] http://news.stanford.edu/news/2012/december/rover-mars-phobos-122812.html. Palo Alto: Stanford Report, 2012 December 28.

12. H.B. Keller. Numerical Solution of Bifurcation and Nonlinear Eigenvalue Problems. Applications of Bifurcation Theory. New York City: Academic Press, 1977.

13. E.L. Allgower, K. Georg (1990). Introduction to Numerical Continuation Methods. Classics in Applied Mathematics, 45. Philadelphia: SIAM, 2003.

14. J.J. Masdemont. High Order Expansions of Invariant Manifolds of Libration Point Orbits with Applications to Mission Design. Dynamical Systems: An International Journal, 20(1), pp. 59-113 (2004).

15. X.Y. Hou, L. Liu. On Motions Around the Collinear Libration Points in the ER3BP. Monthly Notices of the Royal Astronomical Society, 415, pp. 3552-3560 (2011).

16. J. Stoer, R. Bulirsch. Introduction to Numerical Analysis. New York City: Springer-Verlag, 1983.

17. J. Moser. On the Generalisation of a Theorem of Lyapunov. Communications on Pure and Applied Mathematics, 11, pp. 257-271 (1958).

18. G. Gómez, J.M. Mondelo. The Dynamics around the Collinear Equilibrium Points of the RTBP. Physica D, 157(4), pp. 283-321 (2001).

19. E. Kolemen, N.J. Kasdin, P. Gurfil. Multiple Poincaré Sections Method for Finding the Quasiperiodic Orbits of the Restricted Three-Body Problem. Celestial Mechanics and Dynamical Astronomy, 112, pp. 47-74 (2012).

20. Z. Olikara, D.J. Scheeres. Numerical Methods for Computing Quasi-Periodic Orbits and their Stability in the Restricted Three-Body Problem. 1st IAA-AAS Conference on Dynamics and Control of Space Systems, Porto, 2012 March 19-21.

21. A. Jorba. Numerical Computation of the Normal Behavior of Invariant Curves of n-Dimensional Maps. Nonlinearity, 14, pp. 943-976 (2001).

22. R.A. Adomaitis. The Trouble with Spurious Eigenvalues. International Journal of Bifurcation and Chaos, 17(4), pp. 1375-1381 (2007).

23. G. Gómez, G., A. Jorba, J.J. Masdemont, C. Simó. Study Refinement of Semi-Analytical Halo Orbit Theory. Final Report ESOC Contract. Barcelona, 1991.

24. D.L. Richardson. Analytic Construction of Periodic Orbits about the Collinear Points. Celestial Mechanics, 22, pp. 241-253 (1980).

25. B.F. Chao, D.P. Rubincam. The Gravitational Field of Phobos. Geophysical Research Letters, 16(8), pp. 859-862 (1989).

26. T.C. Duxbury, J.D. Callahan. Pole and Prime Meridian Expressions for Phobos and Deimos. The Astronomical Journal, 86(11), pp. 1722-1727 (1981).

27. W.M. Kaula. Theory of Satellite Geodesy. Waltham: Blaisdell, 1966.

28. K. Willner. The Martian Moon Phobos. A Geodetic Analysis of its Motion, Orientation, Shape, and Physical Parameters. Ph.D Thesis. Technische Universität Berlin, 2009.

29. R.M. Brannon. Curvilinear Analysis in an Euclidian Space. UNM Supplemental Book Draft. Albuquerque: University of New Mexico, 2004.

30. V. Szebehely. Theory of Orbits: The Restricted Problem of Three Bodies. New York City: Academic Press, 1967.

31. S. Campagnola, M. Lo, P. Newton. Subregion of Motion and Elliptic Halo Orbits in the Elliptic Restricted Three-Body Problem. 8th AIAA/AAS Space Flight Mechanics Meeting, Glaveston, 2008 January 27-31.

32. R. Broucke. Stability of Periodic Orbits in the Elliptic Restricted Three-Body Problem. AIAA Journal, 7(6), pp. 1003-1009 (1969).

33. J.J Masdemont, J.M. Mondelo. Notes for the Numerical and Analytical Techniques Lectures. Advanced Topics in Astrodynamics Summer Course, Barcelona, 2004. 